\title{
On the Solution of Nonlinear Time-Fractional Generalized Burgers Equation by Homotopy Analysis Method and Modified Trial Equation Method
}

\author{
Haci Mehmet Baskonus, Hasan Bulut, and Yusuf Pandir
}

\begin{abstract}
In this paper, we have executed the Homotopy Analysis Method and Modified Trial Equation Method which has newly been submitted to the literature for obtaining analytical solution of the nonlinear time-fractional generalized Burgers equation occuring in various areas of physics, chemistry, applied sciences, applied mathematics such as modeling of gas dynamics and traffic flow. Then, we have formed a table which includes numerical conclusions for time-fractional generalized Burgers equation. Finally, we have obtained the 2D and 3D surfaces by means of programming language Mathematica 9 in order to interpret in the sense of physical phenomena for analytical solution and approximate solution which have been obtained.
\end{abstract}

Index Terms-Nonlinear time-fractional generalized Burgers equation, homotopy analysis method, modified trial equation method.

\section{INTRODUCTION}

In the last three decades, Fractional Differential Equations and Systems (FDEs, FDES) have investigated keen interests among mathematicians and research scientists, for their analytical and numerical solutions [1]-[15]. To date, methodological studies have been shown not limited to, the numerical difference Differential Transform Method (DTM), the Homotopy Analysis and Homotopy Perturbations Methods (HAM, HPM), the Sumudu Transform Method (STM) and Variational Iteration Method (VIM) used to achieve solutions for various proposed Fractional Differential Equations and Systems.

Liu has introduced to the literature Trial Equation Method for solving nonlinear differential equation [16]-[18]. Gurefe has developed this approach as a Extended Trial equation method [19]. In this paper, we have modified this technique as a Modified Trial equation method (MTEM) for solving nonlinear fractional differential equations [20]-[22].

After applying the MTEM to the nonlinear time-fractional generalized Burgers equation, and producing related surfaces of graphics by means of the symbolic Algebra package, Mathematica Release 7, should we obtain the closer numerical result to analytical solution than by implementing other methods, we would say that HAM is a preferable method of choice for its accuracy on these equations. So, to the best of our knowledge, the modified trial equation method

Manuscript received March 5, 2014; revised May 7, 2014.

The authors are with the Department of Mathematics, Faculty of Science, Firat University, Elazig, Turkey (e-mail: hbulut@firat.edu.tr, yusufpandir@gmail.com, hmbaskonus@gmail.com). has not been widely applied for studying the invariance properties of fractional PDEs.

In this research, we consider nonlinear time-fractional generalized Burgers equation described as following and occur in different areas in mathematical physics;

$$
\frac{\partial^{\alpha} u}{\partial t^{\alpha}}-u_{x x}-\beta u^{p} u_{x}=0
$$

where $0<\alpha \leq 1, p>0$.

The purpose of this paper is to obtain numerical solutions of the nonlinear time-fractional generalized Burgers equation with fractional order by using HAM [21].

\section{Fractional CAlculus Basic Definitions, AND HOMOTOPY ANALYSIS METHOD PROPERTIES}

First of all, we recall some fundamental properties of fractional calculus, and then show the main prospects of the HAM and MTEM. We take into account the HAM and MTEM technique below, for solving nonlinear time-fractional generalized Burgers equation with fractional order [23].

\section{A. Preliminaries}

In this chapter, we recall some definitions and properties of the fractional calculus theory in the sense of Riemann -Liouville derivative proposed by Jumarie. Let $f:[0,1] \rightarrow \Re$ be a continuous function and $\alpha \in(0,1)$. The Jumarie modified fractional derivative of order $\alpha$ and $f$ may be defined by expression of as follows [23]:

$$
D_{x}^{\alpha} f(x)= \begin{cases}\frac{1}{\Gamma(-\alpha)} \int_{0}^{x}(x-\xi)^{-\alpha-1}[f(\xi)-f(0)] d \xi, & \alpha<0, \\ \frac{1}{\Gamma(1-\alpha)} \frac{d}{d x} \int_{0}^{x}(x-\xi)^{-\alpha}[f(\xi)-f(0)] d \xi, & 0<\alpha<1, \\ \left(f^{(n)}(\xi)\right)^{\alpha-n} & , n \leq \alpha \leq n+1, n \geq 1 .\end{cases}
$$

As well as this equality, we may recall the fractional Riemann-Liouville derivative which is used in this study as following [23]:

$$
\begin{aligned}
& D_{x}^{\alpha} k=0 \\
& D_{x}^{\alpha} x^{\mu}= \begin{cases}0, & \mu \leq \alpha-1, \\
\frac{\Gamma(\mu+1)}{\Gamma(\mu-\alpha+1)} x^{\mu-x}, & \mu>\alpha-1 .\end{cases}
\end{aligned}
$$




\section{B. Modified Trial Equation Method (MTEM)}

In this paper, a new trial equation method will be given. In order to apply this method to fractional nonlinear partial differential equations, we consider the following steps.

Step 1. We consider time fractional partial differential equation in two variables and a dependent variable $u$ and take the wave transformation

$$
\begin{aligned}
& P\left(u, D_{t}^{\alpha} u, u_{x}, u_{x x}, u_{x x x}, \cdots\right)=0, \\
& u(x, t)=u(\eta), \quad \eta=k x-\frac{\lambda t^{\alpha}}{\Gamma(1+\alpha)},
\end{aligned}
$$

where $\lambda \neq 0$. Substituting (5) into (4) yields a nonlinear ordinary differential equation

$$
N\left(u, u^{\prime}, u^{\prime \prime}, u^{\prime \prime \prime}, \cdots\right)=0
$$

Step 2. Take trial equation as follows:

$$
u^{\prime}=\frac{F(u)}{G(u)}=\frac{\sum_{i=0}^{n} a_{i} u^{i}}{\sum_{j=0}^{l} b_{j} u^{j}}=\frac{a_{0}+a_{1} u+a_{2} u^{2}+\cdots+a_{n} u^{n}}{b_{0}+b_{1} u+b_{2} u^{2}+\cdots+b_{l} u^{l}}
$$

And

$$
u^{\prime \prime}=\frac{F(u)\left(F^{\prime}(u) G(u)-F(u) G^{\prime}(u)\right)}{G^{3}(u)},
$$

where $F(u)$ and $G(u)$ are polynomials. Substituting above relations into (6) yields an equation of polynomial $\Omega(u)$ of $u$ :

$$
\Omega(u)=\rho_{s} u^{s}+\cdots+\rho_{1} u+\rho_{0}=0 .
$$

According to the balance principle, we can get a relation of $n$ and $l$. We can compute some values of $n$ and $l$.

Step 3. Let the coefficients of $\Omega(u)$ all be zero will yield an algebraic equations system:

$$
\rho_{i}=0, \quad i=0, \cdots, s .
$$

Solving this system, we will specify the values of $a_{0}, \cdots, a_{n}$ and $b_{0}, \cdots, b_{l}$.

Step 4. Reduce (5) to the elementary integral form

$$
\pm\left(\mu-\mu_{0}\right)=\int \frac{G(u)}{F(u)} d u .
$$

Using a complete discrimination system for polynomial to classify the roots of $F(u)$, we solve (11) with the help of Mathematica 9 and classify the exact solutions to (6). In addition, we can write the exact traveling wave solutions of (4). For a better interpretations of results obtained in this way, we plotted 3D surfaces of analytical and approximate solution by taking into consideration suitable parameter.

\section{The Approximate Solution of Time-Fractional GENERALIZED BURGERS EQUATION BY USING HAM}

The analytical solution of nonlinear time-fractional generalized Burgers equation by using MTEM has been obtained as following in [20]:

$$
u(x, t)=\left[\alpha_{1} \pm \frac{\alpha_{1}-\alpha_{2}}{\exp \left[B_{1}\left(x-\lambda_{1} t^{\alpha}\right)\right]-1}\right]^{1 / p},
$$

where $k, p, \beta, \alpha_{1} \neq \alpha_{2}, b_{0}$ are constants and $A_{1}, B_{1}, \lambda_{1}$ are defined by

$$
\begin{aligned}
& A_{1}=-\frac{k(1+p)}{p \beta}, B_{1}=\frac{k\left(\alpha_{1}-\alpha_{2}\right)}{A_{1}}, \\
& \lambda_{1}=\frac{-k \alpha_{1}}{p b_{0} \Gamma[1+\alpha]} .
\end{aligned}
$$

When we regulate the (1) for $p=\beta=1$, we get equation as following:

$$
\frac{\partial^{\alpha} u(x, t)}{\partial t^{\alpha}}-u_{x x}(x, t)-u(x, t) u_{x}(x, t)=0 .
$$

The analytical solution of (14) by using MTEM has been obtained for $p=\beta=1, b_{0}=a_{1}=0.1, a_{2}=0.01$, $\lambda_{1}=0.110326, B_{1}=0.45$ as following:

$$
u(x, t)=0.1+\frac{0.09}{-1+e^{\left(0.45 x-\frac{1}{10 \Gamma[1+\alpha]} t^{\alpha}\right)}} .
$$

The initial condition of (14) is as following:

$$
u(x, 0)=0.1+\frac{0.09}{-1+e^{0.45 x}}
$$

We take into consideration the linear operator

$$
L[\Phi(x, t ; p)]=D_{t}^{\alpha}[\Phi(x, t ; p)],
$$

With the property

$$
L[c]=0 .
$$

We can consider the nonlinear operator $N$ for (14) as following: 


$$
\begin{aligned}
& N[\Phi(x, t ; p)]=\frac{\partial^{\alpha} \Phi(x, t ; p)}{\partial t^{\alpha}} \\
& -\frac{\partial^{2} \Phi(x, t ; p)}{\partial x^{2}}-\Phi(x, t ; p) \frac{\partial \Phi(x, t ; p)}{\partial x}
\end{aligned}
$$

Therefore, we construct the zero-order deformation equation as follows:

$$
\begin{aligned}
& (1-p) L\left[\Phi(x, t ; p)-u_{0}(x, t)\right] \\
& =p \hbar N[\Phi(x, t ; p)] .
\end{aligned}
$$

Absolutely, if $p$ is $p=0$, this gives us initial condition

$$
\Phi(x, t ; 0)=u_{0}(x, t)=u(x, 0)
$$

And if $p$ is $p=1$, this gives us analytical solution

$$
\Phi(x, t ; 1)=u(x, t)
$$

When we expand the solution function of $\Phi(x, t ; p)$ to the tylor series, we obtain as following:

$$
\Phi(x, t ; p)=u_{0}(x, t)+\sum_{m=1}^{\infty} u_{m}(x, t) p^{m},
$$

where

$$
u_{m}(x, t)=\left.\frac{1}{m !} \frac{\partial^{m} \Phi(x, t ; p)}{\partial p^{m}}\right|_{p=0}
$$

If the auxiliary linear operator, the initial condition and the auxiliary parameter $\hbar$ are properly chosen, the above series converges at $p=1$, and one can have

$$
u(x, t)=u_{0}(x, t)+\sum_{m=1}^{\infty} u_{m}(x, t)
$$

When we perform (20) $m$ times by differentiating with respect to $p$, we obtain $m$ th-order deformation equation

$$
L\left[u_{m}(x, t)-\chi_{m} u_{m-1}(x, t)\right]=\hbar R_{m}\left[\vec{u}_{m-1}(x, t)\right],
$$

where $R_{m}\left[\vec{u}_{m-1}(x, t)\right]$ is defined by

$$
\begin{aligned}
R_{m}\left[\vec{u}_{m-1}(x, t)\right]= & \frac{\partial^{\alpha} u_{m-1}(x, t)}{\partial t^{\alpha}}-\frac{\partial^{2} u_{m-1}(x, t)}{\partial x^{2}} \\
& -\sum_{n=0}^{m-1} u_{n}(x, t) \frac{\partial u_{m-1-n}(x, t)}{\partial x}
\end{aligned}
$$

And

$$
\chi_{m}= \begin{cases}0, & m \leq 1 \\ 1, & m>1\end{cases}
$$

The solution of $m$ th-deformation equation for $m \geq 1$ give rise to

$$
u_{m}(x, t)=\chi_{m} u_{m-1}(x, t)+\hbar J_{t}^{\alpha}\left[R_{m}\left[\vec{u}_{m-1}(x, t)\right]\right] .
$$

When we use the initial condition (16) along with (29), we attain the first three terms of (16) as following

$$
\begin{gathered}
u_{0}(x, 0)=0.1+\frac{0.09}{-1+e^{0.45 x}}, \\
m=1 \Rightarrow u_{1}(x, t)=\chi_{1} u_{0}(x, t)+\hbar J_{t}^{\alpha}\left[R_{1}\left[\vec{u}_{0}(x, t)\right]\right], \\
=\hbar J_{t}^{\alpha}\left[\frac{\partial^{\alpha} u_{0}}{\partial t^{\alpha}}-\frac{\partial^{2} u_{0}}{\partial x^{2}}-\sum_{n=0}^{0} u_{0}(x, t) \frac{\partial u_{0}}{\partial x}\right], \\
u_{1}(x, t)=\frac{891 h t^{\alpha}}{16 \times 10^{6} \alpha \Gamma[\alpha]} C \operatorname{s} c h^{2}\left(\frac{9 x}{400}\right), \\
=u_{1}+\hbar J_{t}^{\alpha}\left[\frac{\partial^{\alpha} u_{1}}{\partial t^{\alpha}}-\frac{\partial^{2} u_{1}}{\partial x^{2}}-\sum_{n=0}^{1} u_{n} \frac{\partial u_{n}}{\partial x}\right], \\
u_{2}(x, t)=\frac{891 h t^{\alpha}}{16 \times 10^{6} \alpha \Gamma[\alpha]} C s c h^{2}\left(\frac{9 x}{400}\right) \\
+\frac{891 \times 4^{-7-\alpha} e^{9 x / 200} h^{2} t^{\alpha}}{9765625 \alpha \Gamma[\alpha] \Gamma[1 / 2+\alpha]}\left(-1+e^{9 x / 200}\right)^{-2} \\
\times\left(99 t^{\alpha} \sqrt{\pi} \operatorname{Coth}\left(\frac{9 x}{400}\right)+625 \times 4^{3+\alpha} \Gamma[1 / 2+\alpha]\right), \\
\vdots
\end{gathered}
$$

Thus, the series solution expressed by the HAM can be written in the form of following:

$$
u(x, t)=u_{0}(x, t)+u_{1}(x, t)+u_{2}(x, t)+\cdots .
$$

Then, we can write the approximate solution obtained by HAM of nonlinear time-fractional generalized Burgers equation as following:

$$
\begin{gathered}
u(x, t)=u_{0}(x, t)+\sum_{m=1}^{\infty} u_{m}(x, t) \\
=u_{0}(x, t)+u_{1}(x, t)+u_{2}(x, t)+\cdots, \\
=0.1+\frac{0.09}{-1+e^{0.45 x}}+\frac{891 h t^{\alpha}}{16 \times 10^{6} \alpha \Gamma[\alpha]} C_{s} c h^{2}\left(\frac{9 x}{400}\right) \\
+u_{2}(x, t)=\frac{891 h t^{\alpha}}{16 \times 10^{6} \alpha \Gamma[\alpha]} C \operatorname{sch}\left(\frac{9 x}{400}\right) \\
+\frac{891 \times 4^{-7-\alpha} e^{9 x / 200} h^{2} t^{\alpha}}{9765625 \alpha \Gamma[\alpha] \Gamma[1 / 2+\alpha]}\left(-1+e^{9 x / 200}\right)^{-2} \\
\times\left(99 t^{\alpha} \sqrt{\pi} \operatorname{Coth}\left(\frac{9 x}{400}\right)+625 \times 4^{3+\alpha} \Gamma[1 / 2+\alpha]\right) .
\end{gathered}
$$

The 2D and 3D surfaces of the approximate solution obtained by HAM and analytical solution founded by MTEM for nonlinear time-fractional generalized Burgers equation by using Mathematica 9 programming as following: 

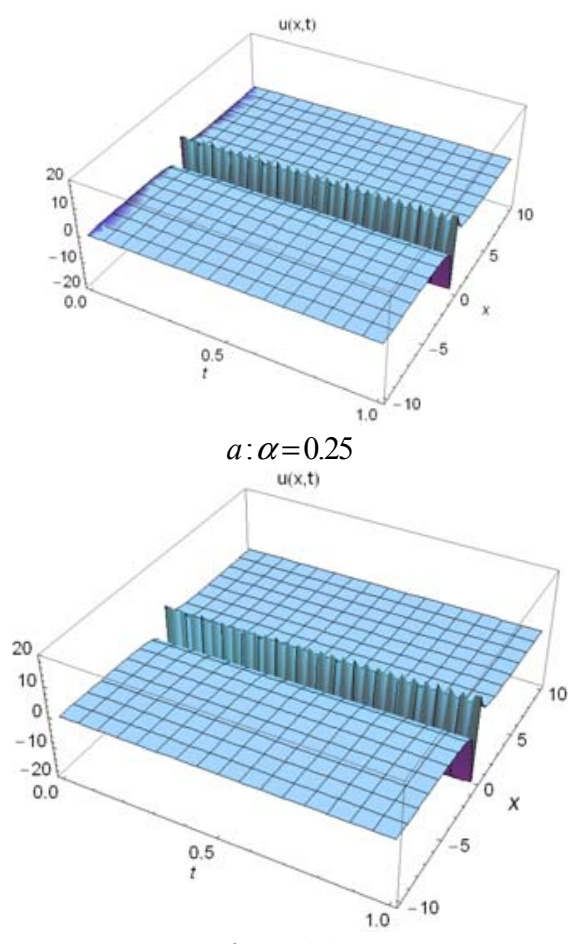

$b: \alpha=0.95$

Fig. 1. The 3D surfaces of (15) analytical solution obtained by means MTEM

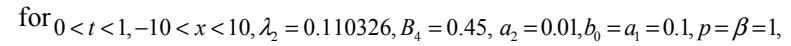

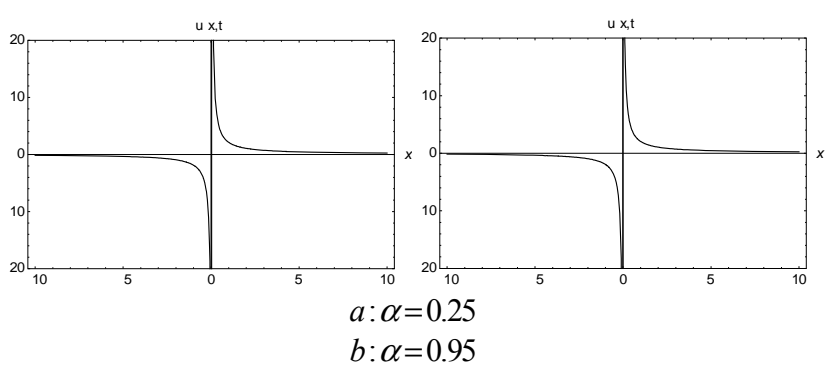

Fig. 2. The 2D surfaces of (15) analytical solution obtained by means MTEM

for $t=0.01,-10<x<10, \lambda_{2}=0.110326, B_{4}=0.45, a_{2}=0.01, b_{0}=a_{1}=0.1, p=\beta=1$.
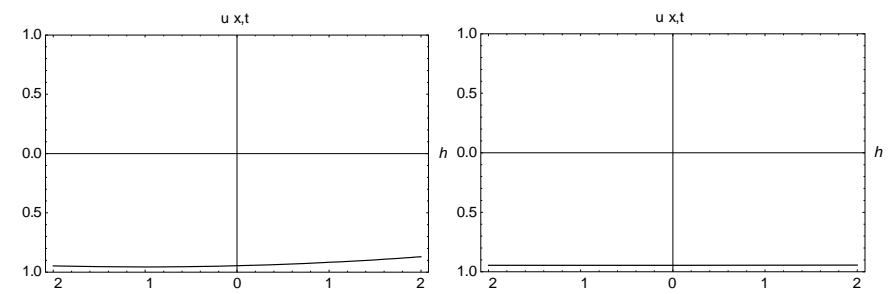

$a: \alpha=0.25 b: \alpha=0.95$

Fig. 3. The 2D surfaces of $h$ being (33) obtained solution by means HAM when $t=0.01, x=-2,-2<h<2$.

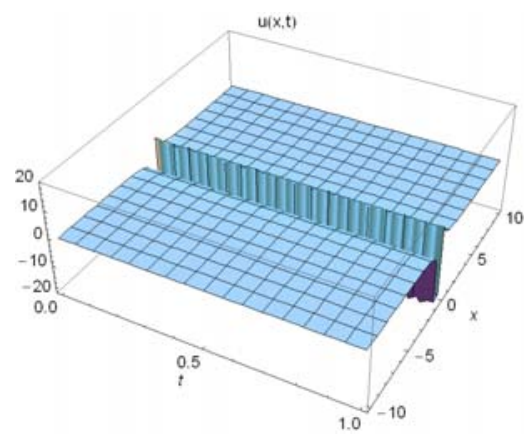

$a: \alpha=0.25$

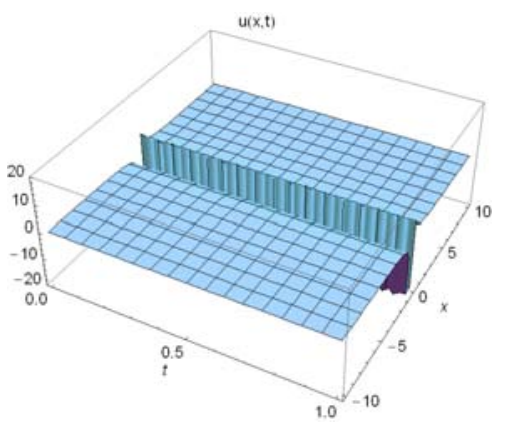

$b: \alpha=0.95$

Fig. 4. The 3D surfaces of (33) approximate solution obtained by means HAM when $-10<x<10,0<t<1, h=-1 / 120$,

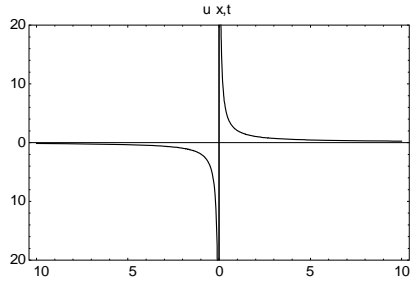

$a: \alpha=0.25$

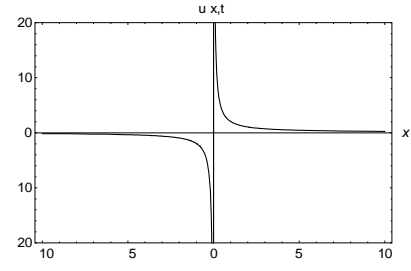

$b: \alpha=0.95$
Fig. 5. The 2D surfaces of (33) approximate solution obtained by means

HAM when $t=0.01,-10<x<10, h=-1 / 120$,

Remark 1:

The solution (33) obtained by using the Homotopy Analysis method for (14) have been checked by Mathematica Program 9. To our knowledge, these analytical and approximate solutions that we find in this paper have been newly submitted to literature. According to these datas, we can comment that these techniques are very convenient for the solutions by showing Fig. 1, Fig. 2, Fig. 3, Fig. 4, and Fig. 5 .

TABLE I: THE RESUlt OF ERRORS OF (33) APPROXIMATE SOLUTION OBTAINED BY MEANS HAM AND ANALYTICAL SOLUTION FOUNDED BY MTEM FOR $\alpha=0.25, t=0.01, h=-1 / 120,-8<x<12$

\begin{tabular}{|c|c|c|c|}
\hline \multirow{2}{*}{$x$} & \multicolumn{3}{|c|}{$\alpha=0.25$} \\
\cline { 2 - 4 } & $u_{\text {Analiti }}$ & $u_{\text {HAM }}$ & $u_{\text {Analiti }}-u_{\text {HAM }}$ \\
\hline-8 & $-1.96620 \mathrm{E}-1$ & $-1.97704 \mathrm{E}-1$ & $1.08367 \mathrm{E}-3$ \\
-4 & $-4.42037 \mathrm{E}-1$ & $-4.46388 \mathrm{E}-1$ & $4.35126 \mathrm{E}-3$ \\
4 & $5.60736 \mathrm{E}-1$ & $5.56309 \mathrm{E}-1$ & $4.42734 \mathrm{E}-2$ \\
8 & $3.08777 \mathrm{E}-1$ & $3.07684 \mathrm{E}-1$ & $1.09317 \mathrm{E}-3$ \\
12 & $2.26171 \mathrm{E}-1$ & $2.25692 \mathrm{E}-1$ & $4.78683 \mathrm{E}-4$ \\
\hline
\end{tabular}

TABLE II: THE RESUlt OF ERRORS OF (33) APPROXIMATE SOLUTION OBTAINED BY MEANS HAM AND ANALYTICAL SOLUTION FOUNDED BY MTEM FOR $\alpha=0.95, t=0.01, h=-1 / 120,-8<x<12$

\begin{tabular}{|c|c|c|c|}
\hline \multirow{2}{*}{$x$} & \multicolumn{3}{|c|}{$\alpha=0.95$} \\
\cline { 2 - 4 } & $u_{\text {Analitik }}$ & $u_{\text {HAM }}$ & $u_{\text {Analitik }}-u_{\text {HAM }}$ \\
\hline-8 & $-1.97654 \mathrm{E}-1$ & $-1.97694 \mathrm{E}-1$ & $4.00745 \mathrm{E}-5$ \\
\hline-4 & $-4.46189 \mathrm{E}-1$ & $-4.46350 \mathrm{E}-1$ & $1.61574 \mathrm{E}-4$ \\
\hline 4 & $5.56509 \mathrm{E}-1$ & $5.56347 \mathrm{E}-1$ & $1.61678 \mathrm{E}-4$ \\
\hline 8 & $3.07733 \mathrm{E}-1$ & $3.07693 \mathrm{E}-1$ & $4.00874 \mathrm{E}-5$ \\
\hline 12 & $2.25714 \mathrm{E}-1$ & $2.25696 \mathrm{E}-1$ & $1.75776 \mathrm{E}-5$ \\
\hline
\end{tabular}




\section{Remark-2:}

When we take into consideration Table I and Table II, the numerical results of analytical solution and approximate solution are very closer. The numerical errors are very smaller, and therefore, we can underline that these methods very suitable for such a fractional differential equations.

\section{CONCLUSION}

In this paper, firstly, the modified trial equation method has been applied to obtain the analytical solution of nonlinear time-fractional generalized Burgers equation. Secondly, the approximate solution of nonlinear time-fractional generalized Burgers equation by using analytical solution attained by using MTEM has been gained by means of Homotopy analysis method. Finally, after we submitted 2D and 3D surfaces for both solutions, we formed a table including numerical results and errors.

As a result of data obtained and the proposed methods in this study, we can say that they can also be applied to other generalized fractional nonlinear differential equations.

\section{REFERENCES}

[1] K. S. Miller and B. Ross, An Introduction to the Fractional Calculus and Fractional Differential Equations, New York, Wiley, 1993.

[2] A. A. Kilbas, H. M. Srivastava, and J. J. Trujillo, Theory and Applications of Fractional Differential Equations, San Diego, Elsevier, 2006.

[3] I. Podlubny, Fractional Differential Equations, San Diego, Academic Press, 1999.

[4] H. Bulut, H. M. Baskonus, and S. Tuluce, "The solution of wave equations by Sumudu transform method," Journal of Advanced Research in Applied Mathematics, vol. 4, no. 3, pp. 66-72, 2012.

[5] H. Bulut, H. M. Baskonus, and S. Tuluce, "Homotopy perturbation Sumudu transform for heat equations," Mathematics in Engineering, Science and Aerospace, vol. 4, no. 1, pp. 49-60, 2013.

[6] Y. Gurefe, A. Sonmezoglu, and E. Misirli, "Application of the trial equation method for solving some nonlinear evolution equations arising in mathematical physics," Pramana-Journal of Physics, vol. 77, no. 6, pp. 1023-1029, 2011.

[7] Y. Pandir, Y. Gurefe, U. Kadak, and E. Misirli, "Classifications of exact solutions for some nonlinear partial differential equations with generalized evolution," Abstract and Applied Analysis, vol. 2012, pp. $16,2012$.

[8] H. Bulut, H. M. Baskonus, and F. B. M. Belgacem, "The Analytical solutions of some fractional ordinary differential equations by sumudu transform method," Abstract and Applied Analysis, vol. 2013, pp. 6, 2013.

[9] H. Bulut, Y. Pandir, and H. M. Baskonus, "Symmetrical hyperbolic Fibonacci function solutions of generalized fisher $\mathrm{s}$ equation with fractional order," in Proc. AIP Conf., 2013, vol. 1558, no. 1914.

[10] A. Esen, O. Tasbozan, and N. M. Yagmurlu, "Approximate analytical solutions of the fractional sharma-tasso-olver equation using homotopy analysis method and a comparison with other methods," Cankaya University Journal of Science and Engineering, vol. 9, no. 2, pp. 139-147, 2012.

[11] H. A. Zedan and E. E. Adrous, "The application of the homotopy perturbation method and the homotopy analysis method to the generalized Zakharov equations," Abstract and Applied Analysis, vol. 2012, pp. 19, 2012.

[12] S. Liao, "On the homotopy analysis method for nonlinear problems," Applied Mathematics and Computation, vol. 147, no. 2, pp. 499-513, 2004.

[13] S. Liao, "Comparison between the homotopy analysis method and homotopy perturbation method," Applied Mathematics and Computation, vol. 169, no. 2, pp. 1186-1194, 2005.
[14] S. Abbasbandy, "The application of homotopy analysis method to solve a generalized hirota-satsuma coupled KdV equation," Physics Letters A, vol. 361, no. 6, pp. 478-483, 2007.

[15] M. A. Dahab, S. M. Mohamed, and T. A. Nofal, "A one step optimal homotopy analysis method for propagation of harmonic waves in nonlinear generalized magneto thermo elasticity with two relaxation times under influence of rotation," Abstract and Applied Analysis, 2013, pp. 14, 2013.

[16] C. S. Liu, "A new trial equation method and its applications," Communications in Theoretical Physics, vol. 45, no. 3, pp. 395-397, 2006.

[17] C. S. Liu, "Trial equation method to nonlinear evolution equations with rank inhomogeneous: Mathematical discussions and its applications," Communications in Theoretical Physics, vol. 45, no.2, pp. 219-223, 2006

[18] C. H. Liu, "New exact envelope traveling wave solutions of high-order dispersive cubic-quintic nonlinear Schrödinger equation," Communications in Theoretical Physics, vol. 44, no. 5, 2005.

[19] Y. Pandir, Y. Gurefe, and E. Misirli, "The extended trial equation method for some time fractional differential equations," Discrete Dynamics in Nature and Society, vol. 2013, pp. 13, 2013.

[20] H. Bulut, H. M. Baskonus, and Y. Pandir, "The modified trial equation method for fractional wave equation and time fractional generalized burgers equation," Abstract and Applied Analysis, vol. 2013, pp. 1-8, 2013.

[21] Y. Pandir and Y. A. Tandogan, "Exact solutions of the time-fractional Fitzhugh-Nagumo equation," in Proc. AIP Conference, 2013, vol. 1558, pp. 1919-1922.

[22] H. Bulut and Y. Pandir, "Modified trial equation method to the nonlinear fractional sharma-tasso-olever equation," International Journal of Modeling and Optimization, vol. 3, no. 4, pp. 353-357, 2013.

[23] H. M. Baskonus, "The numerical solutions of some linear and nonlinear fractional differential equations," Ph.D. dissertation, Dept. Math., Firat Univ., Elazig, 2014.

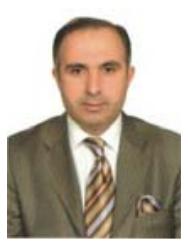

Hasan Bulut is currently an assistant professor of Mathematics in Firat University, Elazig (Turkey). He has published more than 50 articles journals. His research interests include stochastic differential equations, fluid and heat mechanics, finite element method, analytical methods for nonlinear differential equations, mathematical physics, and numerical solutions of the partial differential equations, computer programming. His research papers are published in Abstract and Applied Analysis, Applied Mathematical Sciences, New Word Science Academy and American Institute of Physics.

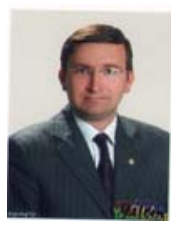

Yusuf Pandir is an assistant professor in the Department of Mathematics at Bozok University; Yozgat (Turkey). He obtained his M.Sc. degree from Celal Bayar University and $\mathrm{Ph} . \mathrm{D}$. degree from Erciyes University. His research interests include fluid mechanics, finite element method, and analytical methods for nonlinear differential equations, mathematical physics, and numerical analysis. His research papers are published in Abstract and Applied Analysis, Applied Mathematical Sciences, International Journal of Physical Sciences, Physica Scripta and Journal of Mathematics.

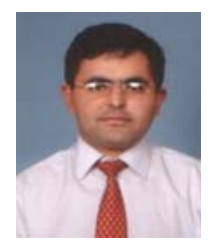

Haci Mehmet Baskonus is currently a lecturer in Tunceli University, Tunceli (Turkey). He obtained his M.Sc. and Ph.D. degree from Firat University. He has published more than 20 articles journals. His research interests include analytical methods for nonlinear differential equations, mathematical physics, and numerical solutions of the partial differential equations, computer programming. His research papers are published in Abstract and Applied Analysis, Applied Mathematical Sciences and American Institute of Physics. 